\title{
CUTLASS Finland radar observations of the ionospheric signatures of flux transfer events and the resulting plasma flows
}

\author{
G. Provan, T. K. Yeoman, S. E. Milan \\ Department of Physics and Astronomy, University of Leicester, University Road, Leicester LE1 7RH, UK
}

Received: 1 December 1997 / Revised: 23 March 1998 / Accepted: 25 March 1998

\begin{abstract}
The CUTLASS Finland radar has been run in a two-beam special scan mode, which offered excellent temporal and spatial information on the flows in the high-latitude ionosphere. A detailed study of one day of this data revealed a convection reversal boundary (CRB) in the CUTLASS field of view (f.o.v) on the dayside, the direction of plasma flow either side of the boundary being typical of a dawn-cell convection pattern. Poleward of the CRB a number of pulsed transients are observed, seemingly moving away from the radar. These transients are identified here as the ionospheric signature of flux transfer events (FTEs). Equatorward of the CRB continuous backscatter was observed, believed to be due to the return flow on closed field lines. The two-beam scan offered a new and innovative opportunity to determine the size and velocity of the ionospheric signatures associated with flux transfer events and the related plasma flow pattern. The transient signature was found to have an azimuthal extent of $1900 \pm 900 \mathrm{~km}$ and an poleward extent of $\sim 250 \mathrm{~km}$. The motion of the transient features was in a predominantly westward azimuthal direction, at a velocity of $7.5 \pm 3 \mathrm{~km}$.
\end{abstract}

Key words. Magnetospheric physics (auroral phenomena; magnetopause, cusp and boundary layers; magnetosphere - ionosphere interaction)

\section{Introduction}

The two-cell convection pattern observed in the highlatitude ionosphere was initially explained by Dungey's (1961) reconnection cycle. Dungey (1961) stated that during periods of southwards interplanetary magnetic

Correspondence to: G. Provan field (IMF), reconnection at the dayside would lead to the creation of open magnetic flux. This open flux would convect over the poles and then be destroyed by reconnection on the nightside. This circulation of flux was originally assumed to be a steady-state phenomenon, with the rate of creation and destruction of open flux being equal at any one instant of time.

In 1972, Russell sketched the ionospheric flows resulting from a non-steady substorm cycle; reconnection on the dayside and in the tail became viewed as two separate time-dependent processes resulting in nonsteady plasma flows in the high-latitude ionosphere (Russell and McPherron, 1973; Siscoe and Huang, 1985; Lockwood et al., 1990). Dayside reconnection events are often categorised as either continuous (quasi-steady) or pulsed (impulsive) (Russell and Elphic, 1978, 1979; Elphic and Russell, 1979). Controversy surrounds the exact nature of these two processes, and the differences, if any, between them. In this study we are primarily interested in the ground-based signatures of impulsive dayside reconnection.

Impulsive dayside reconnection is believed to be the primary mechanism for the transfer of flux from the Earth's magnetosheath to the magnetosphere and episodes of such flux transfer are referred to as flux transfer events (FTEs) (Russell and Elphic, 1978, 1979). Much theoretical work has been developed to explain and model FTEs at the dayside magnetopause and the resulting plasma flows created in the high-latitude ionosphere (Paschmann et al., 1982; Siscoe and Huang, 1985; Cowley, 1984; Southwood, 1985,1987; Lee and $\mathrm{Fu}$, 1985). However, currently there are insufficient high-time and high-spatial resolution observations of FTEs and high-latitude convective flows to verify the various theoretical models.

The first observation of impulsive dayside reconnection was published by Haerendel et al. (1978) using data from the HEOS-2 satellite. Russell and Elphic (1978, 1979), analysed data from the ISEE -1 and -2 satellites and offered a detailed description of flux transfer events 'in which reconnection starts and stops in a matter of 
minutes or less, resulting in the ripping off of flux tubes from the magnetosphere', and offered a theoretical model for the observed magnetic signatures. The FTEs were observed between 9 and 15 MLT and during each observed FTE the magnetosheath field had a southward $B_{z}$ component. Estimated calculations from the satellite data suggested that the ionospheric signature of flux transfer events should have scale size of about $200 \mathrm{~km}$, while the recurrence rate of the FTE events should be 7 $10 \mathrm{~min}$.

The first ground-based observations of flux transfer events were published by Van Eyken et al. (1984) and Goertz et al. (1985). Goertz used data from the STARE radar (a VHF coherent scatter radar sounding the E region ionosphere, Greenwald et al., 1978). They detected a convection boundary in the radar's field of view. Poleward of this boundary the flow was observed to be antisunward, with an occasional significant north-south component. Flows across the convection boundary occurred sporadically in spatially localised regions with scale sizes between $50-300 \mathrm{~km}$, and with a repetition rate of the order of minutes. In one particular event, Goertz et al. (1985) reported observing a poleward moving transient which grew and decayed over a 4 min time interval. Goertz and co-workers (1985) interpreted the transient as the result of newly reconnected flux tubes being pulled poleward by the magnetosheath flow. The event occurred immediately after a turning of the east-west $\left(B_{y}\right)$ component of the IMF from positive to negative.

Rapid velocity transients are commonly observed in the line-of-sight velocity measurements detected by the CUTLASS Finland radar. These features are detected a few hours either side of local noon, at approximate magnetic latitudes of between $70^{\circ}$ to $80^{\circ}$, and are found to be moving away from the radar. They are interpreted here as the ionospheric signatures of FTEs. Conventional theories of FTEs predict that, as newly opened flux tubes are pulled anti-sunward by the magnetosheath flow, ground-based instruments should be able to detect the ionospheric end of the flux tube moving poleward (Goertz et al., 1985), as long as plasma flows are generated which are enhanced relative to the background flow (Southwood, 1987, 1989). We have studied a single interval of data detected by the CUTLASS Finland radar in the pre-noon sector of the 14 August, 1995. During this interval poleward moving velocity transients were observed in the upper range gates of the radar beams, seemingly enhancing the convective plasma flow. The CUTLASS radar was operating in special scan mode on this day to optimise temporal resolution while maintaining good spatial resolution. The scan mode employed has allowed characterisation of the ionospheric signatures of FTEs and the resulting enhanced convective flow in more detail than has been possible in previous studies.

\section{Instrumentation}

The ionospheric convection velocities in this study are provided by the CUTLASS Finland radar. CUTLASS is a bistatic HF coherent radar, with stations in Finland and Iceland, and forms part of the international SuperDARN chain of HF radars (Greenwald et $a l ., 1995)$. Each radar of the system is a frequency agile (8-20 MHz) radar, routinely measuring the line-ofsight (1-o-s) Doppler velocity and spectral width of, and the backscattered power from, ionospheric plasma irregularities. The radars each form 16 beams of azimuthal separation $3.24^{\circ}$. Each beam is gated into 75 range bins, each of length $45 \mathrm{~km}$ in standard operations, when the dwell time for each beam is $7 \mathrm{~s}$, giving a full 16 beam scan, covering $52^{\circ}$ in azimuth and over $3000 \mathrm{~km}$ in range (an area of over $4 \times 10^{6} \mathrm{~km}^{2}$ ), every $2 \mathrm{~min}$. Common-volume data from the two stations can be combined to provide convection velocities perpendicular to the magnetic field, although no common volume backscatter was detected during the interval under study here. During the interval under study here the CUTLASS Finland radar was operating in a non-standard scan mode. In this mode, instead of the usual anti-clockwise sweep through the beams, the radar was confined to sampling on beams 5 and 12 only, i.e. a sequence of $5,12,5,12$ etc. This resulted in a $14 \mathrm{~s}$ time resolution for each beam, with a $45 \mathrm{~km}$ spatial resolution along the beam. At the higher range gates where the data in this study was observed, beam 5 lies to the west of the magnetic meridian while beam 12 lies to the east, there is approximately $34^{\circ}$ between the two beams. This dataset is supplemented by upstream IMF data from the WIND satellite (Lepping et al., 1995).

\section{Data presentation}

\subsection{Radar data}

Figure 1 shows the latitude-time-velocity (LTV) plots for beams 5 and 12 between 0500 and 1010 UT on the 14 August, 1995. The Doppler velocity is colour coded with positive velocities indicating motion towards the radar and negative velocities indicating motion away from the radar, while grey indicates backscatter from the ground. Beam 5 detects ionospheric backscatter between the magnetic latitudes of $72^{\circ}$ to $79^{\circ}$ (altitude corrected geomagnetic, AACGM, co-ordinates, based on PACE geomagnetic co-ordinates, Baker and Wing, 1989), while beam 12 observes backscatter between $73^{\circ}$ and $81^{\circ}$.

Figure 1 illustrates that numerous distinct areas of backscatter are detected by the radar beams, separated by intervals of little or no scatter. The areas of backscatter are observed in general in beam 12 before beam 5 , the first region of backscatter being observed in beam 12 from 0531 until 0653 UT and in beam 5 from 0534 until 0657 UT. During the first half hour of the interval only poleward 1-o-s velocities are observed by beam 12, while beam 5 detects slower equatorward 1-o-s. velocities. From 0600 to 0700 UT, poleward line-ofsight velocities are detected by beam 5 at high ranges but equatorward velocities at lower ranges. The 1-o-s 


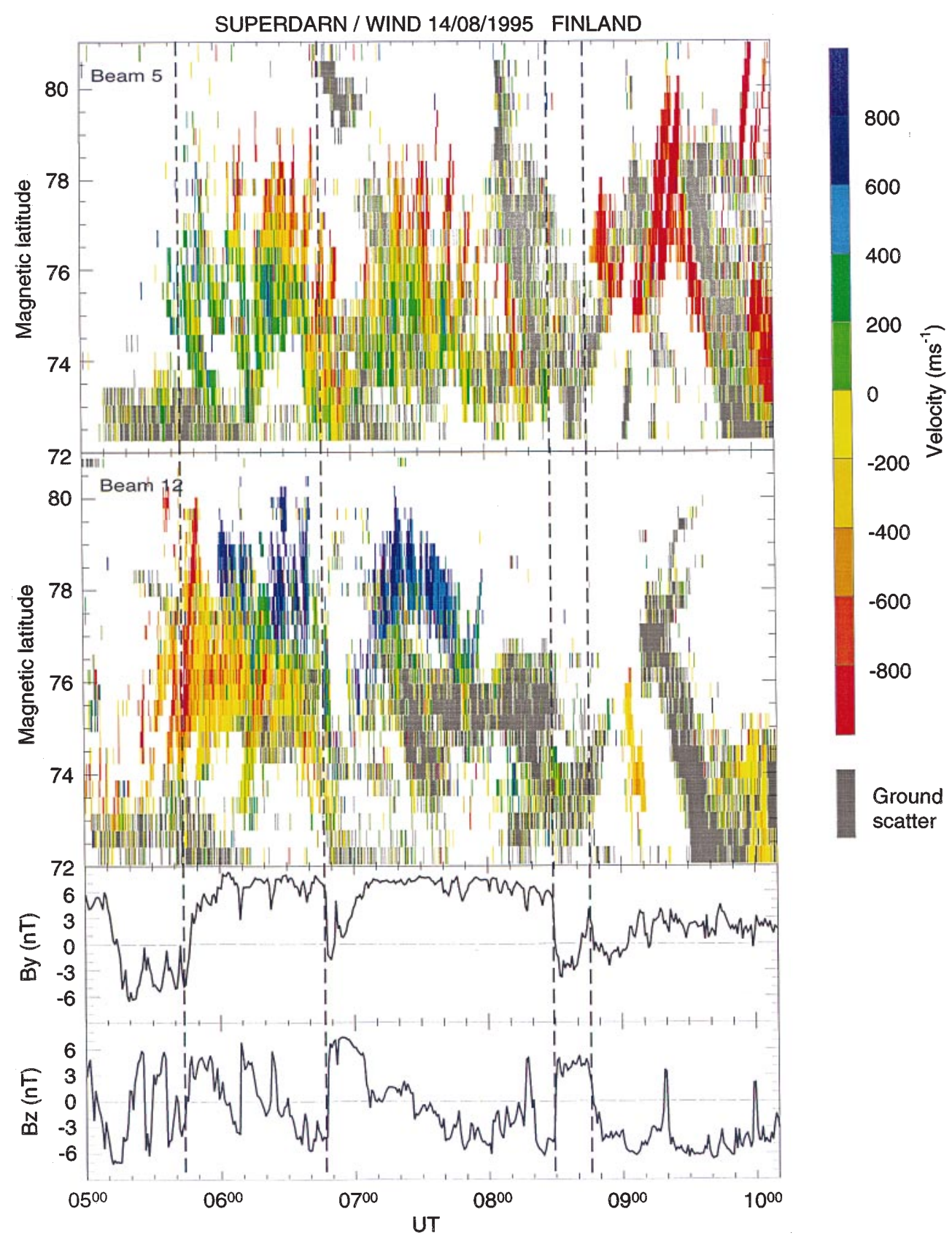

Fig. 1 Latitude-time-velocity (LTV) plots for beams 5 and 12 between 0500 and 1010 on the 14 August 1995. The Doppler velocity is colour-coded with positive velocities indicating motion towards the radar and negative velocities away from the radar. The diagram also shows the $B_{y}$ and $B_{z}$ components of the IMF for the time interval 0500 to 1010 UT, measured by the WIND satellite. The calculated lag time between when the IMF is observed by the satellite and when it impinges on the magnetopause has been added onto the timescale of the IMF plots. Four dashed lines mark the point of significant change of the $B_{y}$ component

velocities observed by beam 12 for this time interval are oppositely directed to beam 5: here the 1-o-s velocity is directed equatorward in the higher range gates and poleward in the lower. From 0700 to 0800 UT, backscatter containing equatorward 1-o-s velocities is seen in the higher ranges of beam 12 which is pointing towards the local noon sector, while beam 5 continues to observe positive 1-o-s velocities at lower ranges and negative $1-0-s$ velocities at higher ranges. From 0850 to 1010 UT beam 5 observes negative l-o-s velocities, with backscatter predominantly located at higher latitudes of between $74.6^{\circ}$ and $81^{\circ}$. Beam 5 is at noon MLT at 1000 UT, whereas, beam 12 is in the early afternoon sector for this interval and does not observe any ionospheric backscatter at all. WIND data presented in panels 3 and 4 of Fig. 1 will be discussed in sec 3.2.
Figure 2 is a schematic representation of the flows detected by the two radar beams during the interval 0600 to 0700 UT. The small arrows depict the direction of the 1-o-s velocity observed by each beam, while the large arrows indicate the direction of convection flows consistent with the observed velocities. The backscatter is detected in the pre-noon sector and indicates westward, anti-Sunward flow at higher ranges and eastward, Sunward flow at lower ranges, with a convection reversal boundary (CRB) in-between. Plasma velocity vectors have been calculated by a beam-swinging technique. The technique assumes that the transient signature is of a scale size such that there is spatial uniformity between the two beams. Considering the angle between the two beams and the magnetic meridian, the 1-o-s velocity observed at the two beams were 


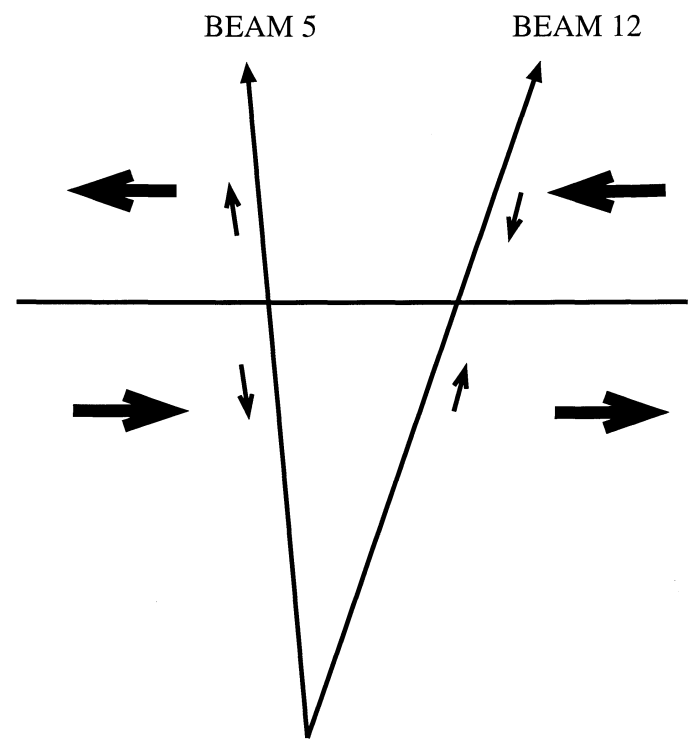

Fig. 2 Schematic representation of the flows detected by the two radar beams during the interval 0600 to 0700 UT. The small arrows depict the direction of 1-o-s velocity observed by each radar beam, while the large arrows point in the direction of convection flows consistent with the observed velocities

combined to gain a value of the poleward and azimuthal velocity components of the plasma flow. Poleward of the $\mathrm{CRB}$, the meridional velocity component of the plasma flow during the transient features is $2 \pm 1 \mathrm{~km} \mathrm{~s}^{-1}$ in a westward azimuthal direction and $0.5 \pm 0.25 \mathrm{~km} \mathrm{~s}^{-1}$ in an equatorward direction.

The convection reversal boundary was detected by one or both radar beams during most of the interval 0600 to 0830 UT. At 0610 UT the equatorward edge of the flow reversal boundary was at a magnetic latitude of $77^{\circ}$ in beams 5 and 12 , by 0650 UT the CRB had moved to a magnetic latitude of $72.5^{\circ}$ in beam 5 and to a magnetic latitude of $74.5^{\circ}$ in beam 12 , this represents an average equatorward motion of the boundary of $180 \mathrm{~m}$ $\mathrm{s}^{-1}$. Little or no backscatter was detected by the two beams between 0650 and 0705 UT. At 0705 UT, the equatorward edge of the flow reversal boundary can be detected at a latitude of $74^{\circ}$ in beam 5 and at a latitude of $75.5^{\circ}$ in beam 12 . The CRB then moves poleward, until 0730, when the flow reversal boundary is at a magnetic latitude of $76^{\circ}$ in beam 5 and $77.5^{\circ}$ in beam 12 . This poleward motion of the flow reversal boundary occurs at an average velocity of $220 \mathrm{~m} \mathrm{~s}^{-1}$. At 0730 , the flow reversal boundary starts moving equatorward again at a velocity of $170 \mathrm{~m} \mathrm{~s}^{-1}$.

Figure 3 presents an LTV plot for the two beams for the time interval 0600 to 0700 UT. Equatorward of the flow reversal boundary backscatter is continuous, the magnitude of the 1-o-s velocity having a quasi-periodic nature. This is especially noticeable in the 1-o-s velocity of beam 12 where the velocity reaches a maximum of between 600 and $800 \mathrm{~m} \mathrm{~s}^{-1}$ with a periodicity of $10 \mathrm{~min}$ or so. Poleward of the CRB both radar beams observe a number of high velocity transients. Some of these transients have been highlighted in Fig. 3, with overplotted black and white lines tracing the motion of individual transients. Three transients which are well defined in both beams have been labelled A, B and C in Fig. 3.

The transients appear as pulsed red stripes in the LTV plot of beam 5 and pulsed blue stripes in the LTV plot of beam 12. The transients are initially observed approximately $2 \mathrm{~min} \pm 30 \mathrm{~s}$ earlier in beam $12 \mathrm{com}$ pared to beam 5 at an estimated $0.5^{\circ}-1^{\circ}$ higher magnetic latitude, in both beams the transients appear to be moving away from the radar. The transients have an average repetition frequency of 7-8 $\mathrm{min}$, and there is no backscatter poleward of the CRB in between these transients. The motion of the transients with respect to the two beams can be determined from a study of the 'gradient' of the transients in the LTV plots, representing their motion to higher range gates with time. The transients have an almost vertical gradient in beam 12 , but a much more oblique gradient in beam 5 . The transients are observed in both beams simultaneously for an estimated two minutes.

Figure 4 shows the latitude-time-spectral-width plot for the two beams between 0600 and 0700 UT. The spectral width is colour-coded with red indicating the largest spectral width values and dark blue the smallest. The plot suggests that the largest spectral width values are detected at range gates furthest away from the radar where the backscatter has a pulsed nature, while nearest range gates where the backscatter is continuous have the smallest spectral widths.

\subsection{WIND data}

Figure 1 shows the $B_{z}$ (north-south) and $B_{y}$ (east-west) components of the IMF for the time interval 0500 to 1010 UT, measured by the WIND satellite. The satellite was located near $\mathrm{X}_{\mathrm{gsm}}=75 \mathrm{R}_{\mathrm{E}}, \mathrm{Y}_{\mathrm{gsm}}=6.9 \mathrm{R}_{\mathrm{E}}$ and $Z_{\mathrm{gsm}}=-7.2 \mathrm{R}_{\mathrm{E}}$. A $13 \pm 1$ min lag-time between when the IMF is observed by the satellite and when it impinges on the subsolar magnetopause has been calculated using the method outlined by Lester et al. (1993). This time lag has been added onto the time scale of the IMF plots, allowing for direct comparison between these and the LTV plots. Four vertical dashed lines mark the times intervals of significant changes in the IMF $B_{y}$ component.

From 0600 to 0830 UT the $B_{y}$ component is generally very stable at about $7 \mathrm{nT}$ with a few negative excursions, while the $B_{z}$ component fluctuates. Comparing the IMF components with the observed l-o-s velocities some clear correlations can be noted. The $B_{y}$ component changes abruptly from $-5 \mathrm{nT}$ to $3 \mathrm{nT}$ at approximately 0545 , while $B_{z}$ turns positive. This corresponds closely to the time of initial backscatter observations by the two radar beams, but no pulsed transients are observed poleward of the CRB until 0600 UT when $B_{y}$ increases to $7 \mathrm{nT}$, while $B_{z}$ turns negative.

At $0647 \mathrm{UT}$, the $B_{y}$ component of the IMF drops from $7 \mathrm{nT}$ to $-2 \mathrm{nT}$ and does not return to its previous value before 0705 , simultaneously the $B_{z}$ component changes from $-4 \mathrm{nT}$ to $7 \mathrm{nT}$ and also remains at this 


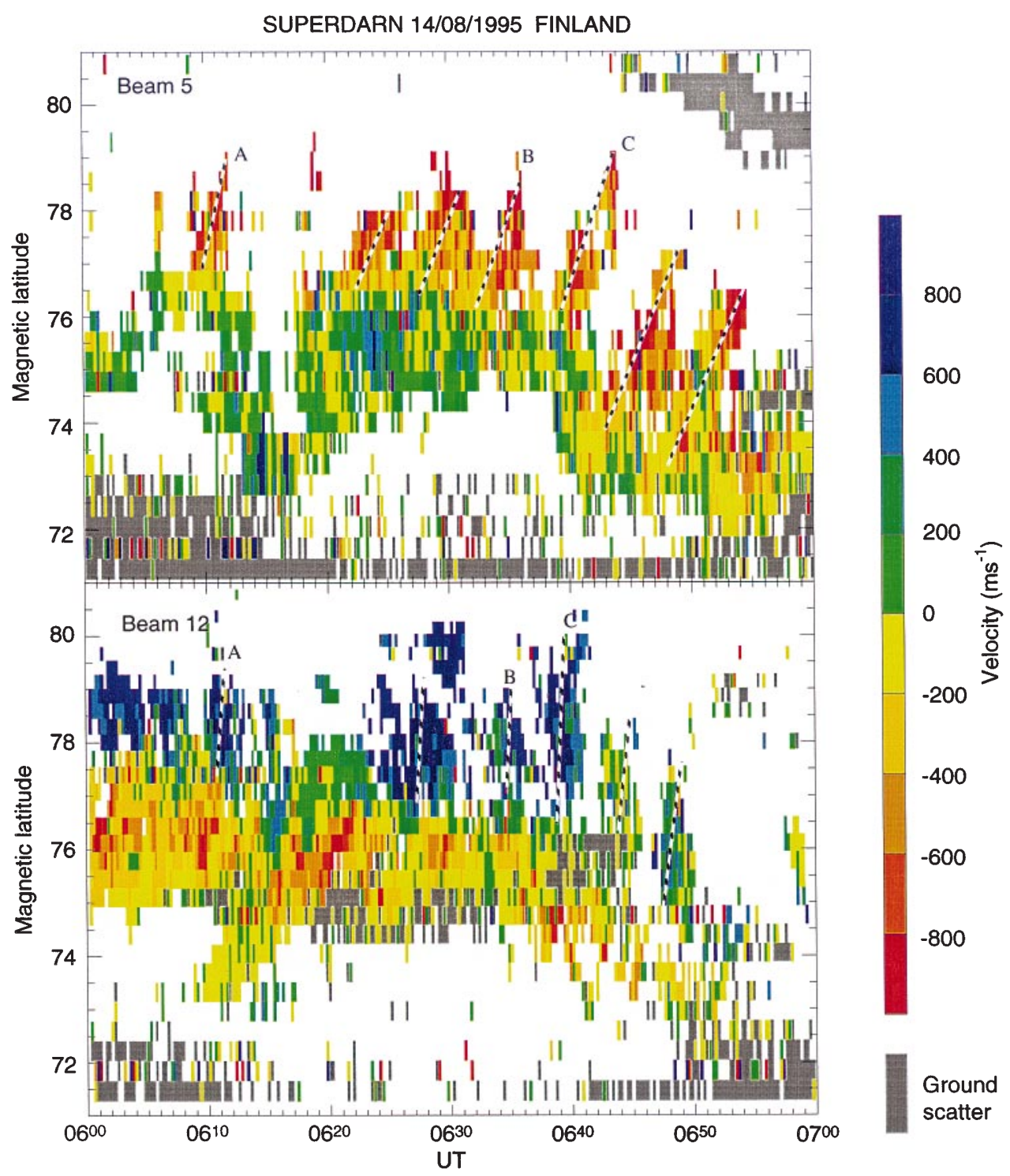

Fig. 3 The LTV plot for beams 5 and 12 for the time interval 0600 to 0700 UT on the 14 August 1995. Overplotted black and white lines trace the motion individual transients. Three transients which are well defined in both beams have been labelled $A, B$ and $C$

value until 0705 . During these $20 \mathrm{~min}$ little or no backscatter is observed by the two radar beams. Pulsed transients are again observed by the two beams between 0705 and 0800 UT. No transient features are then observed by beam 12 after 0800 UT (1230 MLT), while beam 5 continues to observe backscatter until 0830 UT when the $B_{y}$ component of the IMF decreases from $6 \mathrm{nT}$ to $-4 \mathrm{nT}$, while $B_{z}$ increases from $-6 \mathrm{nT}$ to $5 \mathrm{nT}$. No transients are then observed by beam 5 until $0848 \mathrm{UT}$, at this time $B_{y}$ is $4 \mathrm{nT}$ while $B_{z}$ is again negative. Beam 5 then observes backscatter seemingly moving away from the radar, separated by intervals of no backscatter until 1010 (1210 MLT), during this time interval $B_{y}$ is mainly positive and fluctuating around $3 \mathrm{nT}$ while $B_{z}$ is predominantly below $-4 \mathrm{nT}$.

The flow reversal boundary moves equatorward during the time interval 0605-0650 and 0730-0800 UT. During these intervals $B_{y}$ is predominantly stable at around 6-7 nT, while $B_{z}$, although fluctuating between positive and negative values during the first interval, is increasingly negative. From 0710 to 0730 UT the flow reversal boundary is moving in a poleward direction, again $B_{y}$ is approximately $7 \mathrm{nT}$, while $B_{z}$ has low positive values (between 0 and $2 \mathrm{nT}$ ).

\section{Discussion}

A convection reversal boundary is detected in the CUTLASS radar data, poleward of the CRB the plasma flows in an westward (anti-Sunward) direction while equatorward of the CRB the plasma flow is in an eastward (Sunward) direction. Equatorward of the CRB the observation of backscatter is constant, the magnitude of the 1-o-s velocity having a quasi-periodic nature. Poleward of the CRB, pulsed backscatter moving away from the radar is observed with a repetition rate of about $7 \mathrm{~min}$. During the intervals of pulsed backscatter the CRB appears to move to lower latitudes. The direction of plasma flow either side of the CRB is consistent with the radar observing a typical southward IMF dawn convection cell with plasma rotating in an 


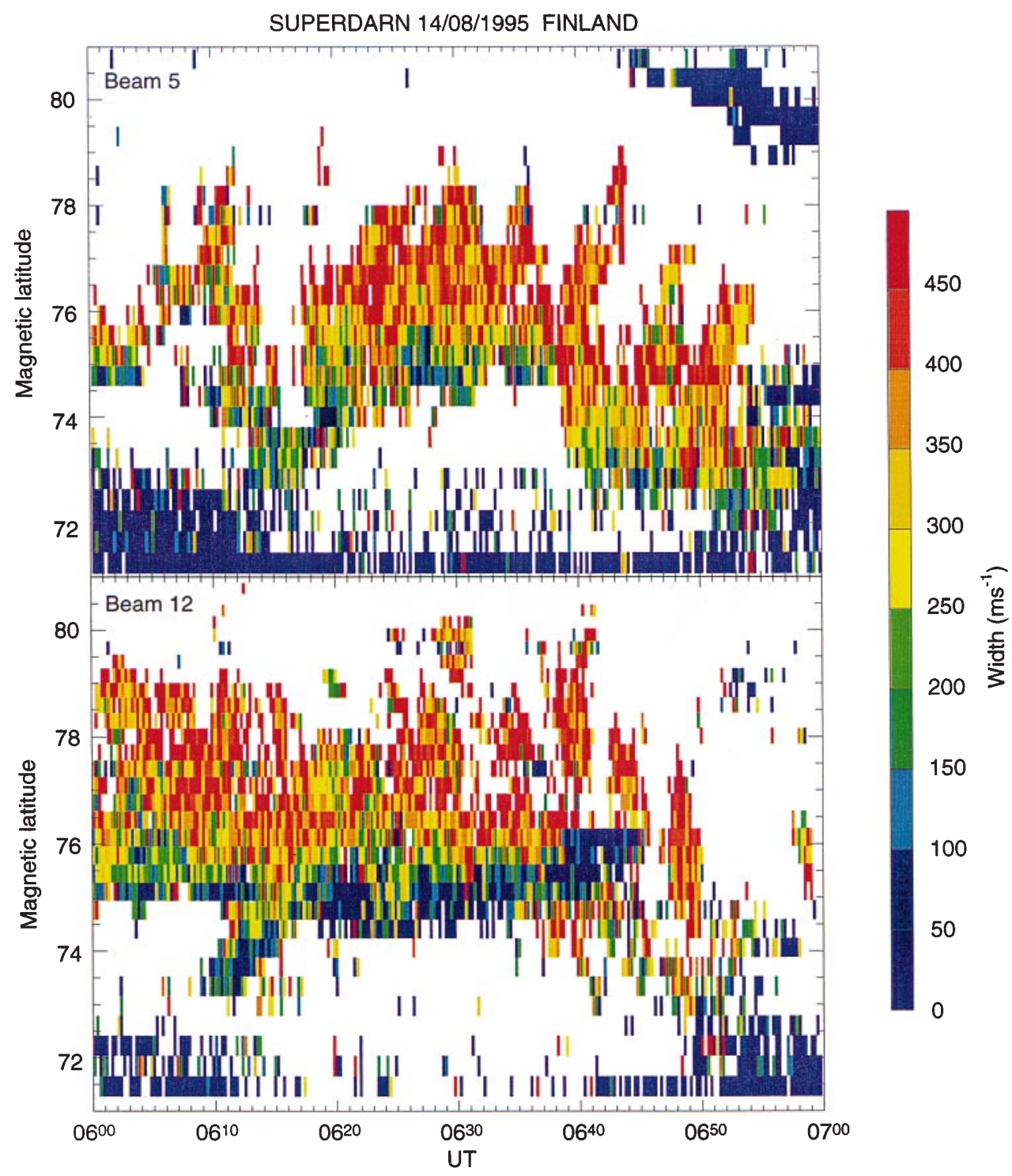

Fig. 4 Latitude-time-spectralwidth plot for beams 5 and 12 between 0600 and 0700 on the 14 August 1995. The spectral width is colour-coded with red velocities indicating the largest spectral width and dark blue representing the smallest spectral width anti-clockwise direction (Dungey, 1961). The convection cell is observed for approximatley four and a half hours of univeral time, from 0530 UT when it is first detected by beam 5, until 1010 UT, this corresponds to radar coverage of approximately seven hours of magnetic local time. When the convection cell is first observed the far ranges of beam 5 are located at $\sim 0730$ MLT, while the far ranges of beam 12 are observing at approximately 1000 MLT. At 1000 the far ranges of beam 5 are located at $\sim 1200$ MLT, and the far ranges of beam 12 are located at $\sim 1430$ MLT.

During the intervals when transients were observed by the radar beams, they had an approximate average recurrence rate of 7 to $8 \mathrm{~min}$, this is close to the average recurrence rate of flux transfer events first reported by Rijnbeek et al. (1984). It is our belief that the transients observed poleward of the CRB are the ionospheric signature of flux transfer events at the magnetopause boundary. Continuous backscatter observed equatorward of the CRB is due to return flow on closed field lines, the periodic enhancements of the 1-o-s velocity being a response to the transient nature of reconnection at the subsolar point. Poleward of this radar observations are not continuous, with the inferred dawn-cell convection pattern only detected by the radar when the conditions are met for the generation of $\mathrm{F}$ region irregularities from which the radar backscatters. The excellent spatial information offered by the two-beam radar scan allows a detailed investigation of the spatial and temporal characteristics of the ionospheric signatures of FTEs. This interpretation of the radar data is presented in more detail later.

Individual transients are initially observed on average $\sim 2$ min \pm 30 s earlier in beam 12 than in beam 5 , and are detected in both beams simultaneously for $\sim 2 \mathrm{~min}$. Beam 12 is at a later MLT than beam 5 and is therefore closer to local noon during the earlier part of the interval studied. The transients are initially observed approximately $1^{\circ}$ of magnetic latitude nearer the pole in beam 12 compared to beam 5 . At the latitudes that the transients are observed the two beams have an azimuthal separation of between 750 and $950 \mathrm{~km}$. As the 
transient signature takes $2 \mathrm{~min} \pm 30 \mathrm{~s}$ to travel from one beam to the next, the transients have an azimuthal velocity component of $7.5 \pm 3 \mathrm{~km} \mathrm{~s}-1$ and a velocity component towards the radar of $0.8 \pm 0.4 \mathrm{~km} \mathrm{~s}^{-1}$. The transient signatures extend $1900 \pm 900 \mathrm{~km}$ in an azimuthal direction and $\sim 250 \mathrm{~km}$ in a poleward direction (between 5-6 range gates). The estimated average magnetic latitude at which the transients were observed was $77^{\circ}$, one hour of MLT at a latitude of $77^{\circ}$ is equal to approximately $375 \mathrm{~km}$ in an azimuthal direction. So the transient signature covers approximately $5 \pm 2.5$ hours of MLT. Previous work by Elphic et al. (1990), using data from the ISEE 1 and 2 satellites in conjunction with data from the EISCAT radars and photometers and an all-sky camera, have inferred that the ionospheric footprint of FTEs extended between $400-1000 \mathrm{~km}$ in the east-west direction and between 100-200 km in the north-south direction. Comparing these results with the size of the ionospheric footprint of FTEs observed by us, we can see that although the scale sizes observed by Elphic et al. (1990) are smaller in both the north-south and east-west extent, the two sets of results are in agreement within the large error ranges given.

As the transient signature can be observed by both beams for several minutes, we have used a beamswinging technique, which assumes spatial uniformity between the two beams, to calculate that the plasma flow during the transient features have an azimuthal velocity component. The equatorward component of the plasma flow velocity during the transient features is $0.5 \pm 0.25 \mathrm{~km} \mathrm{~s}^{-1}$, while the azimuthal component is $2 \pm 1 \mathrm{~km} \mathrm{~s}^{-1}$. The error range for these velocities is so large mainly because of difficulty in tracing the position of the HF radar beams at very high latitude. Since the two beams have are separated by $34^{\circ}$ it is unlikely that the region between them is totally uniform, leading to further uncertainty in the measured velocities. However, this is an inherent problem of the beam-swinging technique and it is impossible to estimate the scale of these errors. The equatorward velocity component of the transients is equal to the equatorward velocity of the plasma flow, within the given error range. The azimuthal velocity component of the transient would appear to be somewhat greater than the azimuthal velocity component of the plasma flow.

The 'gradient' that the transients make in the latitude-time-velocity (LTV) plots of the two beams, suggest that the transients move almost perpendicular to beam 12 while moving away from the radar in beam 5 . Taking these mentioned factors into account, it is clear that the transient features move in a predominantly westward azimuthal direction, being detected in beam 12 before beam 5. Between the two beams the transients have a small velocity component towards the radar, however it would appear that the poleward component of motion of the transients changes direction, as the transients appear to be moving away from the radar when observed in beam 5 . The motion of the transients seem best described as being along a curved trajectory on open field lines, possibly consistent with motion along a notional $\mathrm{L}$ shell.
Figure 5 is a polar plot showing the position of the radar beams with respect to geomagnetic latitude at 0630 UT. Overlaid is a schematic illustration of our interpretation of the motion of the flux transfer event and related plasma convection flow. The elliptical 'blob' represents the patch of irregularities probably associated with soft particle precipitation on newly opened field lines created by the flux transfer event. The black line marks the open/closed field line boundary, while the grey-hatched zone represents the region of continuous backscatter observations. The newly opened field lines initialise the convective flow of plasma. Equatorward of the opened/closed field line boundary, steady energetic electron precipitation associated with the low-latitude boundary layer is known to produce continuous backscatter (Baker et al., 1995), allowing us to observe continuously the modulated velocity within the convective plasma flow. Poleward of the boundary, the area of newly opened flux will move predominantly azimuthally, in a direction almost perpendicular to beam 12 . Precipitation from this patch will create irregularities from which the radar can backscatter, highlighting the background westward convective flow associated with plasma being dragged anti-Sunward. The net effect of a period of flux transfer events is the motion of the open/ closed field line boundary to lower latitude. The direction of motion of the transient signature, the creation of return flow on closed field line and the motion of the open/closed field line boundary to lower latitudes is very similar to the model of dayside reconnection suggested by Cowley et al. (1992) and Cowley and Lockwood (1992). Similar observations of high-latitude plasma flow variations accompanied by bursts of return flow at lower latitudes have previously

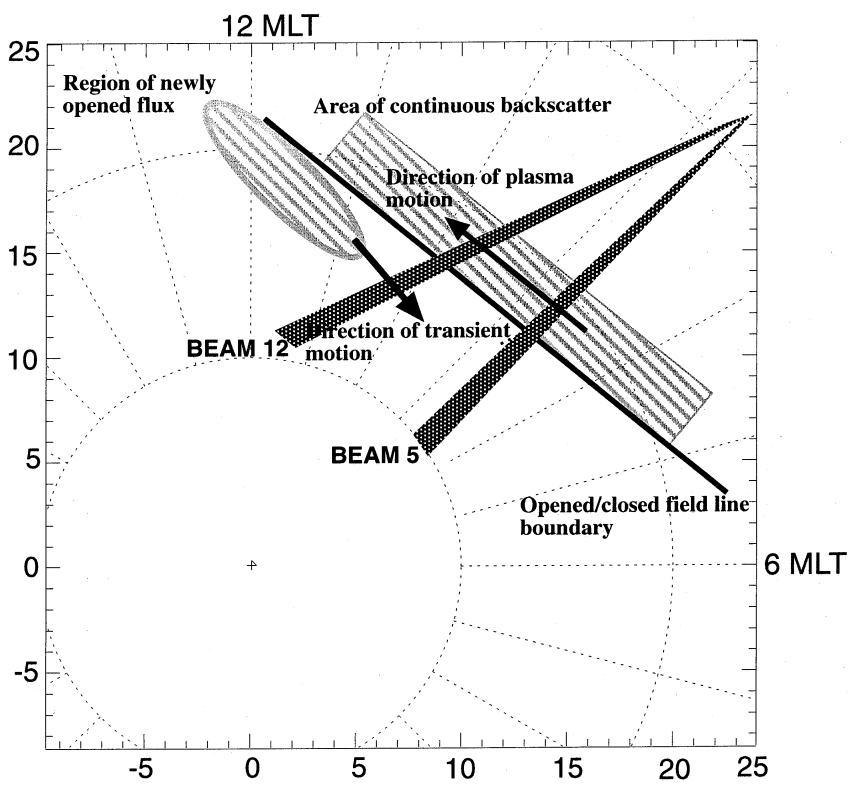

Fig. 5 Polar plot showing the position of the radar beams with respect to geomagnetic latitude at 0630 UT. Overlaid is a schematic of the most likely motion of the poleward moving transients in the polar cap and the region of transient velocity response in the return flow 
been observed by Moen et al. (1995), using satellite data combined with optical observations and data from the EISCAT radar.

Baker et al. $(1990,1995)$ have identified the HF radar signature of the low-latitude boundary layer (LLBL) and the cusp, using the spectral width distribution of backscattered l-o-s velocities observed by the Halley HF radar and low-latitude particle precipitation data from the DMSP spacecraft. They stated that the distribution of spectral widths in the cusp is generally of a Gaussian form with a peak at $220 \mathrm{~m} \mathrm{~s}^{-1}$, while the distribution of spectral widths in the LLBL is more likely to be an exponential distribution, with the peak of the distribution occurring at $50 \mathrm{~m} \mathrm{~s}^{-1}$. Figure 6 shows a plot of the spectral width distribution of the positive and negative line-of-sight velocities for beams 5 and 12 between 0500 and 0800 UT. At lower range gates positive (negative) velocities observed by beam 5 (beam 12) have a spectral width distribution with a modal value of approximately $300(250) \mathrm{m} \mathrm{s}^{-1}$, while at higher range gates beam 5 (beam 12) negative (positive) velocities have a distribution with a modal value of about $350(350) \mathrm{m} \mathrm{s}^{-1}$.

Comparing Baker's results with the spectral width distribution of the 1-o-s velocities observed by the CUTLASS radar between 0500 and 0830 UT, both poleward and equatorward of the flow reversal boundary, it can be seen that the distributions are remarkably similar. Both radar beams observed a spectral width distribution of a general Gaussian form poleward of the flow reversal boundary. The form of the spectral width distributions observed equatorward of the CRB for both beams was similar to the shape of the spectral width distribution Baker et al. (1995) identified in the LLBL region, although the modal values of the spectral width distributions observed by the CUTLASS radar are somewhat higher than those detected by Baker et al. (1995). The shape of the spectral width distributions are so similar that we feel confident that, based on the Baker definition, we can identify the polar cap boundary in the pre-noon sector in the CUTLASS f.o.v, with anti-

Finland: Spectral width distributions, 14/08/1995, 0500 to 0800 UT
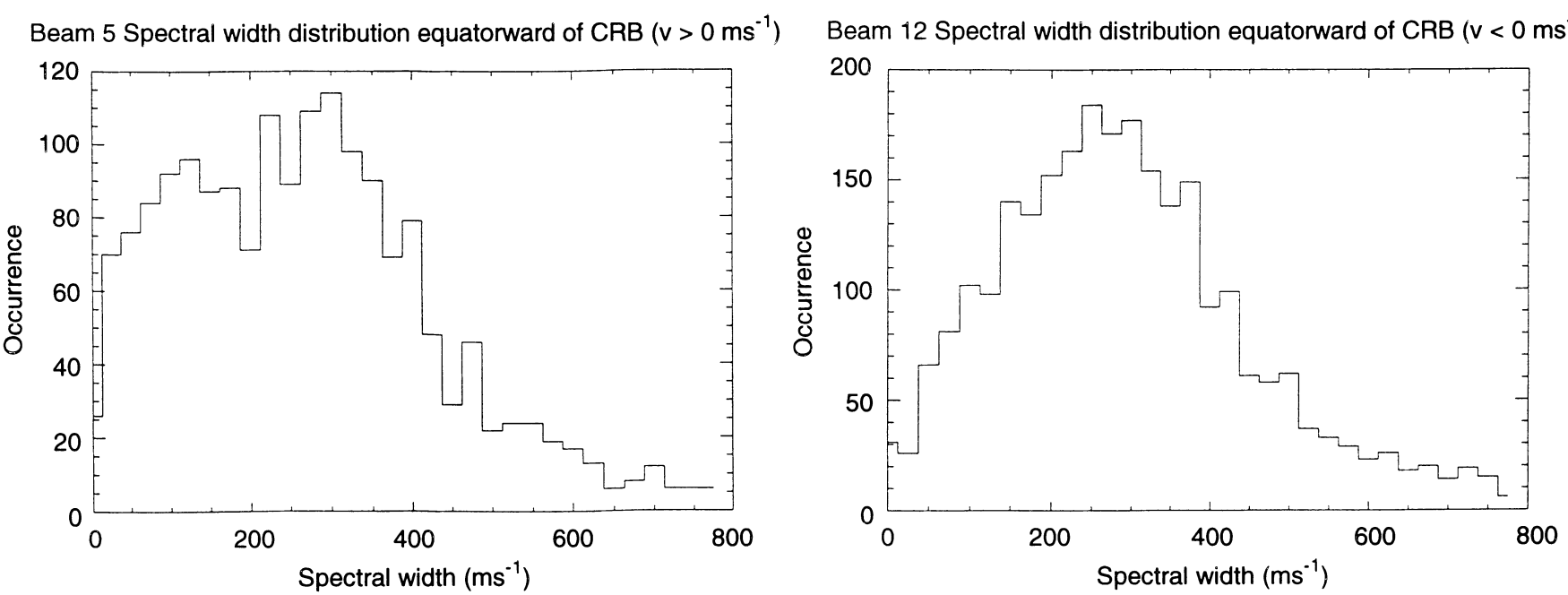

Beam 5 Spectral width distribution poleward of CRB $\left(v<0 \mathrm{~ms}^{-1}\right)$ Beam 12 Spectral width distribution poleward of CRB $\left(v>0 \mathrm{~ms}^{-1}\right)$
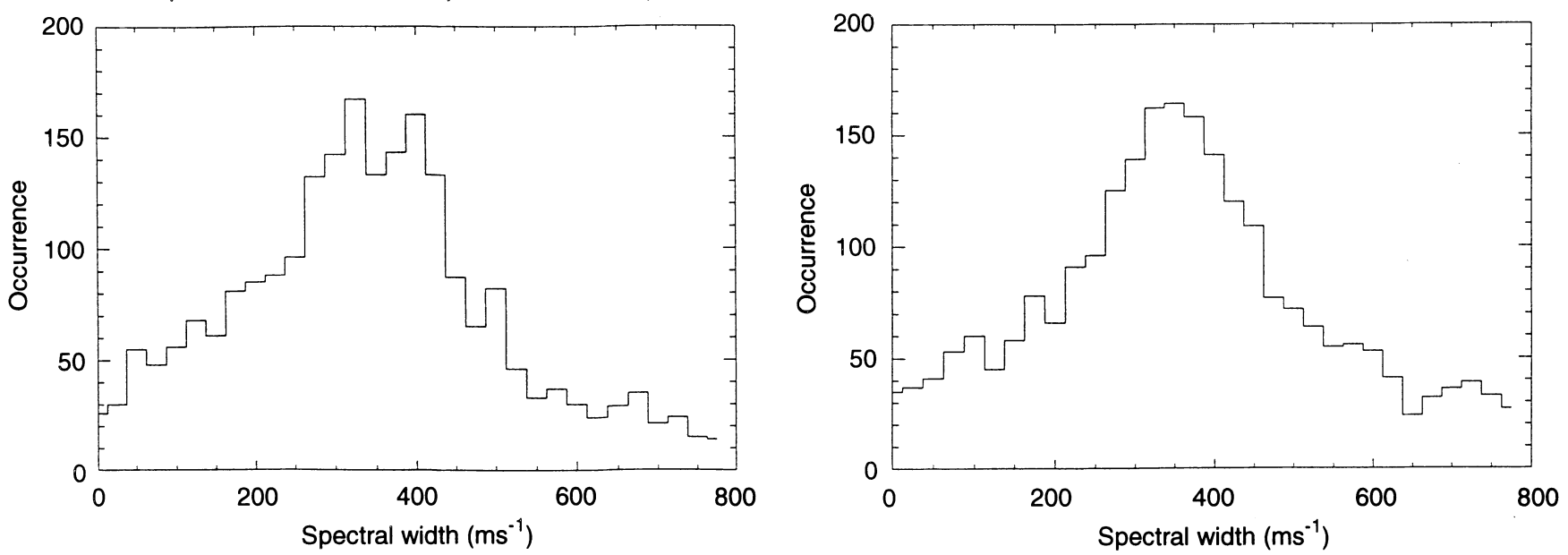

Fig. 6 The spectral width distribution of the positive and negative line-of-sight velocities for beams 5 and 12 between 0500 and 0800 UT. In general the negative (positive) velocities are detected poleward (equatorward) of the CRB in beam 5 and are oppositely directed in beam 12 
Sunward flows on open field lines within the polar cap and Sunward flows, at lower latitudes on closed field lines. The FTEs add open flux to the polar cap which enhances the overall convective flow.

Drawing conclusions about the effect of the IMF $B_{y}$ and $B_{z}$ components on the observed FTE signatures and the background convection pattern is complicated in this case study, as a change in one component is often accompanied by a variation in the other. This is the case, for example, in the interval 0645 to $0705 \mathrm{UT}$, when a sudden decline in the amount of backscatter detected, is accompanied by a drop in the $B_{y}$ component of the IMF (from $7 \mathrm{nT}$ to $-2 \mathrm{nT}$ ) and also by a sudden increase in the $B_{z}$ component of the IMF ( from $-4 \mathrm{nT}$ to $7 \mathrm{nT}$ ). However, since the changes in the $B_{y}$ and $B_{z}$ components correspond so well with a variation in the radar observations, this is confirmation that any error in the calculated lag-time of the IMF between the WIND satellite and the Earth is within the given margins.

During the first two intervals, when poleward moving transients are detected, the $B_{y}$ component is predominantly above 6-7 nT (0600-0645 and 0705-0830 UT) while the $B_{z}$ component fluctuates greatly between positive and negative values. During the third interval of transient observations ( 0845 to 1010 UT) the $B_{y}$ component fluctuates around $3 \mathrm{nT}$ while $B_{z}$ is mainly below $4 \mathrm{nT}$. The motion of the FTEs in a westwards azimuthal direction can be explained by the SvalgaardMansurov effect (Smith and Lockwood, 1996, and references therein), where a positive IMF $B_{y}$ component in the Northern Hemisphere exerts a curvature force on magnetic field lines, forcing them to move towards dawn. No FTE signatures are observed during extended intervals of high positive $B_{z}\left(0645-0705 \mathrm{UT}, B_{z}=7 \mathrm{nT}\right)$, but dayside reconnection does occur during intervals of low, positive $B_{z}\left(0705-0730 \mathrm{UT}, B_{z}=0-2 \mathrm{nT}\right)$.

The motion of the flow reversal boundary adheres to conventional reconnection theory (Dungey, 1961, 1963) and can be explained by the model of dayside reconnection proposed by Cowley et al. (1992) and Cowley and Lockwood (1992). During intervals of predominantly negative $B_{z}$ (0605-0647 UT and 0730-0800 UT), the flow reversal boundary moves equatorward. While during intervals of positive $B_{z}(0710-0730)$ the flow reversal boundary moves poleward. This is consistent with newly opened flux being added to the polar cap due to dayside reconnection during intervals of negative $B_{z}$, causing the polar cap boundary to expand, whilst during intervals of positive $B_{z}$, reconnection on the nightside leads to the destruction of open flux, and the polar cap shrinks.

\subsection{Comparison with previous studies}

The FTEs were created during a period of mainly positive $B_{y}$. The ionospheric signature of the FTEs is similar to the theoretical model predicted by Cowley et al. $(1991,1992)$ and Cowley and Lockwood (1992) for a period of positive $B_{y}$ in the northern hemisphere. Cowley and co-workers proposed that the FTEs would create a convection pattern in the ionosphere at the front of the polar cap boundary. Initially, the convection pattern would move azimuthally at an approximate velocity of $2 \mathrm{~km} \mathrm{~s}^{-1}$ for six minutes, resulting in a total east-west displacement of about $700 \mathrm{~km}$. As the system moved towards its equilibrium configuration, the mainly azimuthal motion of the patch will give way to poleward motion and a twin vortical flow will be excited over the auroral zone. The time scale for the excitation and decay of this flow pattern is predicted to be about 10-15 min.

The time scale, direction of motion and velocity of the ionospheric signature of the flux transfer events observed by the CUTLASS radar correspond well to the theoretical model proposed by Cowley and co-workers (Cowley et al., 1991, 1992; Cowley and Lockwood, 1992). The CUTLASS radar also observed return flow on closed field lines, which is central to the conceptual framework postulated by Cowley and colleagues. There are a number of points to make about the CUTLASS observations in view of theoretical model developed by Cowley and co-workers. First of all, backscatter associated with newly opened magnetic flux can only be observed poleward of the CRB, this is probably because the patch of newly opened backscatter is observed in the pre-noon sector, only after it has moved across the opened/closed field line boundary. Secondly, the transient signature observed by the CUTLASS radar has an azimuthal extent of $1900 \pm 900 \mathrm{~km}$ and a poleward extent of about $250 \mathrm{~km}$. This is more than twice the size of the transient signature predicted by Cowley. However, as the radar is observing the signature in the prenoon sector, it is likely that the signature has been 'stretched' by the magnetosheath flow by this stage, as also predicted by the Cowley model.

Poleward of the CRB the plasma velocity is $2 \pm 1 \mathrm{~km} \mathrm{~s}^{-1}$ in a westward azimuthal direction and $0.5 \pm 0.25 \mathrm{~km} \mathrm{~s}^{-1}$ in an equatorward direction. Sandholt et al. (1992) observed periodic auroral events at the midday polar cap boundary, seemingly the ionospheric signature of flux transfer events. These events moved eastward near the noon meridian with a velocity of $1.5 \mathrm{~km} \mathrm{~s}^{-1}$. This is within the error range of the azimuthal component of the plasma flow observed poleward of the CRB by CUTLASS. The transients observed by the CUTLASS radar moved with a velocity of $7.5 \pm 3 \mathrm{~km} \mathrm{~s}^{-1}$ in an azimuthal direction and with a velocity of $0.8 \pm 0.4 \mathrm{~km} \mathrm{~s}^{-1}$ towards the radar. Cowley and colleagues (Cowley et al., 1991, 1992; Cowley and Lockwood, 1992) predicted that the velocity of the area of newly opened flux would be the same as the velocity of the convective flow. The CUTLASS observations indicate that the transients' velocity appears to be larger than the velocity of the plasma flow, especially in the azimuthal direction. It is possible that the transients are actually moving with the same velocity as the convective flow and that any discrepancy between the two measured velocities is due to the size of the error margins being underestimated. Alternatively, it may be that the observed transients are not the ionospheric footprints of stable, newly opened field lines but are created by precipitation due to active reconnection still taking place 
at the magnetopause boundary. The observed velocity of the transients would then not be the plasma velocity but a phase velocity in the ionosphere. However at the present time it is not possible to definitively verify whether the observed transients are signatures of newly opened field lines or the signatures of field lines on which reconnection is still active.

Pinnock et al. (1995) and Rodger and Pinnock (1997) used data from the Halley PACE HF radar to study the ionospheric signature of flux transfer events, specifically the early evolution of these transients. They reported observations of poleward moving high velocity features very similar to those observed by the CUTLASS radar and identified them as flux transfer events. They concentrated especially on the early evolution of the transient signature in relation to the theory of FTEs. The transient features increased the 1-o-s Doppler velocity by up to $0.5 \mathrm{~km} \mathrm{~s}^{-1}$ and were superimposed upon a background poleward flow of about $300 \mathrm{~m} \mathrm{~s}^{-1}$. The transients themselves were observed to have broad spectral widths and were first observed within regions of low spectral width (identified as the LLBL), up to three degrees equatorward of a region of high spectral width (identified as the cusp), into which they propagated over a $10 \mathrm{~min}$ period. The boundary between the low and high spectral width regions was moving equatorward at a velocity of $82 \mathrm{~m} \mathrm{~s}^{-1}$. No IMF data was available for the interval studied.

Both the CUTLASS and Halley radars observe transient signatures, indicative of FTE activity, moving away from the radar and superimposed upon background convection flow, but there are some fundamental differences between the CUTLASS and Halley results. Firstly, whereas the transients signatures indicative of open field lines are observed by the Halley radar a few degrees equatorward of the CRB and propagate to a few degrees poleward of it, the transients observed by the CUTLASS radar are only detected poleward of the CRB. Secondly, whereas the background convective flows observed by Halley were directed poleward either side of the boundary between high and low spectral widths, the flows detected by CUTLASS were directed anti-Sunward on the poleward side of the CRB and Sunward on the equatorward side. Thirdly, Pinnock et al. (1995) suggested that the transients were moving poleward, whereas observations made by the CUTLASS radar shows that the motion of the transients is predominantly azimuthal.

In comparing the present observations with those made by Pinnock et al. (1995) we did consider whether the quasi-periodic nature of the magnitude of the 1-o-s velocity observed equatorward of the $\mathrm{CRB}$, especially in beam 12, was due to the regions of open flux propagating poleward. However, studying the spectral widths of these regions of increased l-o-s velocity in Fig. 4, we can conclude that these features do not have an increased spectral width compared to the surrounding area and are most likely caused by the enhancement of the return flow due to the addition of open flux. They are thus most probably a response to the FTE rather than a direct signature of it.
It is possible to explain the ionospheric signatures of FTEs made by the Halley and CUTLASS radars within the same theoretical framework. The observations by Pinnock et al. (1995) were made between 10 and 13 MLT, close to local noon, while CUTLASS made its observations in the pre-noon sector. Therefore, Halley would observe the area of newly opened flux when it had just been added onto the polar cap boundary but was still equatorward of the main CRB, whereas we have already mentioned that CUTLASS did not observe the regions of newly opened flux until after they crossed the opened/closed field line boundary. As Halley is located in the noon sector, it is likely that the radar is looking down the convection throat at antiSunward flow. The radar would thus observe the boundary between high and low spectral widths, with the 1-o-s velocities directed in the same direction either side of the boundary. Similar transient signatures moving in a poleward direction only, are observed by CUTLASS beam 5 between 0850 and 1010 UT in Fig. 1. During this period beam 5 is observing data between $\sim 11-12$ MLT. The transients observed in beam 5 during this period is then a result of the radar looking down the convection throat at anti-Sunward flow. Finally, the transients observed by the Halley radar seemed to be moving poleward, due to the fact that the radar was only observing in a high-time resolution mode on one beam. The CUTLASS radar was observing on two beams. When observing the transients in each beam separately, they appear to be moving poleward. However, combining the data from both beams shows that the transients were moving in a predominant azimuthal direction.

\section{Summary and conclusion}

The CUTLASS radar, run in a two-beam high-time resolution mode, has been able to offer unrivalled spatial and temporal information on plasma flows in the highlatitude ionosphere. The time-averaged radar signatures are typical of a dawn-cell convection pattern. Equatorward of the CRB backscatter is observed continuously with the magnitude of the 1-o-s velocity having a quasiperiodic nature, while poleward of the boundary pulsed transients are observed. We believe that these pulsed transients are the ionospheric signature of flux transfer events. The two-beam mode allows us to make the following observations on FTEs and the related flows in the ionosphere:

1. The transients had a recurrence rate of between 7 to 8 minutes.

2. The ionospheric signature of the FTEs have an approximate azimuthal extent of $1900 \pm 900 \mathrm{~km}$ and an estimated poleward extent of $\sim 250 \mathrm{~km}$.

3. The motion of the transients is predominantly azimuthal, the transients move in an westward azimuthal direction with a velocity of $7.5 \pm 3 \mathrm{~km}$ $\mathrm{s}^{-1}$ and towards the radar with a velocity of $0.8 \pm 0.4 \mathrm{~km} \mathrm{~s}^{-1}$. 
4. Poleward of the CRB plasma velocity is $2 \pm 1 \mathrm{~km}$ $\mathrm{s}^{-1}$ in a westward azimuthal direction and $0.5 \pm$ $0.25 \mathrm{~km} \mathrm{~s}^{-1}$ in an equatorward direction.

5. Although the velocity of the transients is almost equal to the velocity of the plasma flow within the large error range given, it would appear that the velocity of the transients is somewhat larger than the velocity of the convective flow. It is possible that we are not actually observing the ionospheric footprints of newly opened field lines, but precipitation from regions of still-active reconnection at the magnetopause boundary.

6. The magnitude of the 1-o-s velocity equatorward of the $\mathrm{CRB}$ has a quasi-periodic nature, reaching a maximum value every $10 \mathrm{~min}$ or so. This period is approximately the same as the recurrence rate of the FTEs. It would appear that the addition of open flux at the subsolar point leads to an detectable enhancement in the magnitude of the return flow

7. Using a spectral width criterion for identifying the HF signature of the cusp and LLBL (Baker et al., 1995), we can identify the region of pulsed antiSunward convecting backscatter as mapping to open field lines and the region of continuous Sunward convecting backscatter as mapping to closed field lines.

8. Our results suggest that the ionospheric signature of FTE were only detected by the CUTLASS radar during intervals when the $B_{y}$ component of the IMF was greater than $3 \mathrm{nT}$, and while the $B_{z}$ component was less than $2 \mathrm{nT}$. The problem of deconvolving the separate effects of the $B_{y}$ and $B_{z}$ components of the IMF on the observed FTEs and background convection pattern, means that these results will have to be viewed as initial ones, further research on this topic will follow.

9. Further work using greater temporal and spatial resolution measurements from the CUTLASS radar will we hope in the future be able to make unambiguous measurements of the velocities of plasma convection and FTE signatures in the high-latitude ionosphere.

Acknowledgements. Topical Editor D. Alcayde' thanks J. Moen and R. C. Elphic for their help in evaluating this paper.

\section{References}

Baker, K. B., and S. Wing, A new magnetic coordinate system for conjugate studies at high latitudes, J. Geophys. Res., 94, 91399142, 1989.

Baker, K. B., R. A. Greenwald, J. M. Ruohoniemi, J. R. Dudeney, M. Pinnock, P. T. Newell, M. E. Greenspan, and C.-I. Meng, Simultaneous HF-radar and DMSP observations of the cusp, Geophys. Res. Lett., 17, 1869, 1990.

Baker, K. B., J. R. Dudeney, R. A. Greenwald, M. Pinnock, P. T. Newell, A. S. Rodger, N. Mattin, and C.-I. Meng, HF radar signatures of the cusp and low-latitude boundary layer, J. Geophys. Res., 100, 7671, 1995.

Cowley, S. W. H., Solar wind control of magnetospheric convection, in Achievement of the International Magnetospheric Study, IMS, p. 483, Noorwijk, Netherlands, 1984.
Cowley, S. W. H., M. P. Freeman, M. Lockwood, and M. F. Smith, The ionospheric signature of flux transfer events, in Cluster Dayside Polar Cusp, ESA SP-330., 105, 1991.

Cowley, S. W. H., and M. Lockwood, Excitation and decay of solar wind-driven flows in the magnetosphere-ionosphere system, Ann. Geophysicae, 10, 103, 1992.

Cowley, S. W. H., J. P. Morreli, M. P. Freeman, M. Lockwood, and M. F. Smith, Excitation and decay of flows in the magnetosphere-ionosphere system due to magnetic reconnection at the dayside magnetopause and in the geomagnetic tail, in Proc. International Conference on Substorms, ESA SP-335, p. 117, 1992.

Dungey, J. W., Interplanetary field and the auroral zones, Phys. Res. Lett., 647-648, 1961.

Dungey, J. W., The structure of the ionosphere, or adventures in velocity space, in Geophysics: The Earth's Environment, Ed. C. DeWitt, J. Hiebolt and A. Lebeau, p. 526, Gordon and Breach, New York, 1963.

Elphic, R. C., and C. T. Russell, ISEE-1 and -2 observations of the magnetopause, in Magnetospheric Boundary Layers, ESA SP148, 1979.

Elphic, R. C., M. Lockwood, S. W. Cowley, and P. E. Sandholt, Flux transfer events at the magnetopause and in the ionosphere, Geophys. Res. Lett., 17, 2241-2244, 1990.

Goertz, C.K., E. Nielsen, A. Korth, K.-H. Glassmeier, C. Haldoupis, P. Hoeg, and D. Hayward, Observations of a possible signature of flux transfer events, J. Geophys. Res., 90, 4069-4078, 1985.

Greenwald, R. A., W. Weiss, E. Nielsen and N. R. Thomson, STARE a new radar auroral backscatter experiment in northern Scandinavia, Radio Sci., 13, 1021-1039, 1978.

Greenwald, R. A., K. B. Baker, J. R. Dudeney, M. Pinnock, T. B. Jones, E. C. Thomas, J.-P. Villain, J.-C. Cerisier, C. Senior, C. Hanuise, R. D. Hunsucker, G. Sofko, J. Koehler, E. Nielsen, R. Pellinen, A. D. M. Walker, N. Sato, and H. Yamagishi, DARN/SuperDARN: a global view of the dynamics of highlatitude convection, Space Sci. Rev., 71, 761, 1995.

Haerendel, G., G. Paschmann, N. Sckopke, H. Rosenbauer and P. C. Hedgecock, The frontside boundary layer of the magnetopause and the problem of reconnection, J. Geophys. Res., 83, 3195, 1978.

Lee, L. C., and Z. F. Fu, A theory of magnetic flux transfer at the Earth's magnetopause, Geophys. Res. Lett., 12, 105, 1985.

Lester, M., O. de la Beujardière, J. C. Foster, M. P. Freeman, H. Lühr, J. M. Ruohoniemi, W. Swider, The response of the large scale ionospheric convection pattern to changes in the IMF and substorms: results from the SUNDIAL 1987 campaign, Ann. Geophysicae, 11, 556-571, 1993

Lepping, R. P., et al., The WIND magnetic field investigation, Space. Sci. Rev., 71, 207-229, 1995.

Lockwood, M., P. E. Sandholt, A. D. Farmer, S. W. H. Cowley, B. Lybekk and V. N. Davda., Auroral and plasma flow transients at magnetic noon, Planet. Space. Sci., 38, 973-993, 1990.

Moen, J., P. E. Sandholt, M. Lockwood, W. F. Denig, U. P. Løvhaug, B. Lybekk, A. Egeland, D. Opsvik and E. FriisChristensen, Events of enhanced convection and related dayside auroral activity, J. Geophys. Res., 23, 917-934, 1995.

Paschmann, G., G. Haerendel, I. Papamastorakis, N. Sckopke, S. J. Bame, J. T. Gosling, and C. T. Russell, Plasma and magnetic field characteristics of magnetic flux transfer events, J. Geophys. Res., 87, 2157-2168, 1982.

Pinnock, M., A. S. Rodger, J. R. Dudeney, F. Rich, and K. B. Baker, High spatial and temporal resolution observations of the ionospheric cusp, Ann. Geophysicae, 13, 919-925, 1995.

Rijnbeek, R. P., S. W. H. Cowley, D. J. Southwood, and C. T. Russell, A survey of dayside flux transfer events observed by the ISEE-1 and -2 magnetometers, J. Geophys. Res., 89, 786-800, 1984.

Rodger, A. S., and M. Pinnock, The ionospheric response to flux transfer events: the first few minutes, Ann Geophysicae, 15, 685$691,1997$. 
Russell, C. T., The configuration of the magnetopshere, in Critical Problems of Magnetospheric Physics, Ed E.R. Dyer, National Academy of Sciences, Washington, D. C.,1972.

Russell, C. T., and R. L. McPherron, The magnetotail and substorms, Space Sci. Rev., 15, 205, 1973.

Russell, C. T., and R. C. Elphic, Initial ISEE magnetometer results: magnetopause observations, Space Sci. Rev., 22, 681, 1978.

Russell, C. T., and R. C. Elphic, ISEE observations of flux transfer events at the dayside magnetopause, Geophys. Res. Lett., 6, 33, 1979.

Sandholt, P. E., J. Moen, and D. Opsvik, Periodic auroral events at the midday polar cap boundary: implications for solar windmagnetosphere coupling, Geophys. Res. Lett., 19, 1223-1226, 1992.
Siscoe, G. L., and T. S. Huang, Polar cap inflation and deflation, J. Geophys Res, 90, 543-547, 1985.

Smith, M. F., and M. Lockwood, Earth's magnetospheric cusps, Rev. Geophys; 34, 233-260,1996.

Southwood, D. J., Theoretical aspects of ionosphere-magnetopshere, solar wind coupling, in Physics of the Ionosphere-Magnetopshere, Adv. Space. Res., 5, 4, 1985.

Southwood, D. J., The ionospheric signature of flux transfer events, J. Geophys. Res., 92, 3207-3213, 1987.

Southwood, D. J., Magnetopause coupling processes and the ionospheric response: a theoretical perspective, Philos. Trans. R. Soc. London. Ser. A. 328, 79, 1989.

Van Eyken, A. P., H. Risbeth, and M. A. Saunders, Initial observations of plasma convection at invariant latitudes of 70-77 , J. Atmos. Terr. Phys., 46, 635, 1984. 